\title{
Using p-mode excitation rates for probing convection in solar-like stars
}

\author{
F. Kupka, ${ }^{1}$ K. Belkacem, ${ }^{2,3}$ M.-J. Goupil, ${ }^{2}$ and R. Samadi ${ }^{2}$ \\ ${ }^{1}$ Max-Planck-Institute for Astrophysics, Karl-Schwarzschild Str. 1, 85748 Garching, Germany \\ ${ }^{2}$ Observatoire de Paris, LESIA, CNRS UMR 8109, 92195 Meudon, France \\ ${ }^{3}$ Institut d'Astrophysique et de Géophysique de I'Université de Liège, Allée du 6 Août 17, 4000 Liège, \\ Belgium
}

\begin{abstract}
We discuss how the possibility to measure mode excitation rates through means of helio- and asteroseismology has improved our capabilities to test convection models and numerical simulations of surface convection and avoids ambiguities that have limited previous approaches.

Individual Objects: Sun, $\alpha$ Cen A

\section{Probing Stellar Convection Models}

Classical methods used in stellar evolution theory to "calibrate" the mixing length parameter $\alpha$ are based on integral quantities such as radius. However, if considered as an actual probe of convection models including mixing length theory itself such methods remain ambiguous (cf. also the study in Montalbán et al. 2004). Moreover, apart from the case of nearby stars, the basic stellar parameters required to derive these integral quantities can usually not be determined independently from the stellar structure models in which the convection models to be tested are actually used. A common alternative to this approach are tests which require to know physical conditions as a function of optical depth, for instance, the comparison of synthetic Balmer line profiles with observed ones (Gardiner at al. 1999, Heiter et al. 2002). A clear preference in favour of one particular convection model cannot be based on such comparisons either, except for the notion that none of the standard local convection models are able to permit predictions of detailed spectral line profiles for the entire range of A- to Gtype stars. Furthermore, such tests have only limited implications on how reliably convection is modelled inside the star.
\end{abstract}

\section{Seismology as an alternative?}

That restriction does not hold for methods based on helio- and asteroseismology. Convection driven p-mode oscillations directly depend on the physical conditions of layers both near the stellar surface and inside the star. The comparison of predicted to observed p-mode frequencies has become a popular challenge for convection models used in stellar structure theory. Constraints such as the depth of the solar convection zone and the thickness of the region where deviations from an adiabatic temperature gradient occur ("overshooting") or the chemical composition (cf. Houdek 2009) probe convection models also in layers inside the star. Still, this approach can suffer from ambiguities when used to probe the critical superadiabatic layers of stellar convective envelopes, because the same acoustic size of a resonant cavity can originate from either a locally steeper temperature gradient (Basu \& Antia 1995) or a more 
extended envelope due to turbulent pressure and the different efficiency of radiative cooling of a homogeneous (1D) compared to an inhomogeneous (3D) photosphere (Rosenthal et al. 1999). Hence, additional information is needed to remove these ambiguities and probe different properties of a convection model or a numerical simulation of convection.

\section{Probing Convection with Excitation Rates}

Fortunately, there is more information contained in helio- and asteroseismic observations: mode amplitudes and mode widths, which can now be obtained with direct measurements also from COROT (e.g., Appourchaux et al. 2008), and mode life times. Mode excitation due to shear stresses and entropy fluctuations is taken into account in a model by Samadi \& Goupil (2001) which directly links the observed quantities with quantities such as the root mean square vertical velocity and convective flux as a function of depth (for an alternative approach and further references see also Houdek et al. 1999, for development of the physical concept see Gough 1980 and Christensen-Dalsgaard \& Frandsen 1983, for a review Houdek 2006). The model requires eigenfunctions, associated eigenfrequencies, the mean structure, spatial and temporal correlations related to the turbulent kinetic energy and entropy, as well as the filling factor (relative horizontal area covered by regions of upflow) as a function of depth from a numerical simulation or a convection model. Seismological measurements and analyses provide the mode mass, the geometrical height at which modes are measured, the mode line width at half maximum, and the mean square of the mode surface velocity. Note that mode mass and geometrical height need some model input as well. With these data one can compute and compare predicted with observed mode excitation rates.

\section{Results and further progress}

Using this approach Samadi et al. (2006) were able to test several convection models widely used in the literature. Standard solar models (assuming a grey photosphere and a mixing length treatment of convection) underestimate the excitation of solar $\mathrm{p}$ modes by an order of magnitude. Samadi et al. (2006) could demonstrate that although both one-dimensional model atmospheres which account for non-grey radiative transfer and the full spectrum turbulence models as tested by Basu \& Antia (1995) predict much higher mode excitation rates (up to a factor of 3 ), local convection models all fall clearly short of the observationally permitted error range. In earlier work Samadi et al. (2003) had shown that predictions derived from numerical simulations in turn do agree with observed data. Samadi et al. (2006) concluded that a non-local approach to convection modelling was needed which accounts for the asymmetric probability distributions of up- and down-, of hot and cold flows. A new model (Belkacem et al. 2006a) takes these properties into account. The "convection mode with plumes" is based on a model for higher order correlations of velocity and temperature for convective flows by Gryanik \& Hartmann (2002). Though not all the input data for the model can yet be obtained without the use of numerical simulations, the model is a large step forward, since it was shown to reproduce p-mode excitation rates within observational uncertainties (Belkacem et al. 2006b). Recently, it was possible to repeat this work for the case of $\alpha$ Cen A (Samadi et al. 2008). The paper also analyzed the contributions of turbulent pressure, flow asymmetry, the time-correlation between eddies, and entropy fluctuations vs. Reynolds stresses. It was concluded that further a reduction of observational uncertainties (by typically a factor of 3 ) would allow asteroseismology to provide equally powerful tests as helioseismology now does for testing convection models.

Acknowledgments. F.K.'s work was possible thanks to a one month grant provided by the Observatoire de Paris. We are grateful to M.-A. Dupret and F. Baudin for their contributions to this research. 


\section{References}

Appourchaux, T., Michel, E., Auvergne, M., et al. 2008, A\&A, 488, 705

Basu, S., \& Antia, H. M. 1995, ASP Conf. Ser., 76, 649

Belkacem, K., Samadi, R., Goupil, M.-J., \& Kupka, F. 2006a, A\&A, 460, 173

Belkacem, K., Samadi, R., Goupil, M.-J., et al. 2006b, A\&A, 460, 183

Christensen-Dalsgaard, J., \& Frandsen, S. 1983, Solar Physics, 82, 469

Gardiner, R. B., Kupka, F., \& Smalley, B. 1999, A\&A, 347, 876

Gough, D. O. 1980, in "Nonradial and Nonlinear Stellar Pulsation", eds. H. A. Hill and W. A. Dziembowski, Lecture Notes in Physics, 125, 273

Gryanik, V. M., \& Hartmann, J. 2002, J. Atmos. Sci., 59, 2729

Heiter, U., Kupka, F., van 't Veer-Menneret, C., et al. 2002, A\&A, 392, 619

Houdek, G. 2006, in "Beyond the spherical Sun", eds. K. Fletcher and M. Thompson, published on CDROM, ESA SP, 624, 28

Houdek, G., \& Gough, D. O. 2009, CoAst, 159, 27

Houdek, G., Balmforth, N. J., Christensen-Dalsgaard, J., \& Gough, D. O. 1999, A\&A, 351, 582

Montalbán, J., D'Antona, F., Kupka, F., \& Heiter, U. 2004, A\&A, 416, 1081

Rosenthal, C. S., Christensen-Dalsgaard, J., Nordlund, Å, et al. 1999, A\&A, 351, 689

Samadi, R., \& Goupil, M.-J. 2001, A\&A, 370, 136

Samadi, R., Nordlund, Å, Stein, R. F., et al. 2003, A\&A, 404, 1129

Samadi, R., Kupka, F., Goupil, M.-J., et al. 2006, A\&A, 445, 233

Samadi, R., Belkacem, K., Goupil, M.-J., et al. 2008, A\&A, 489, 291

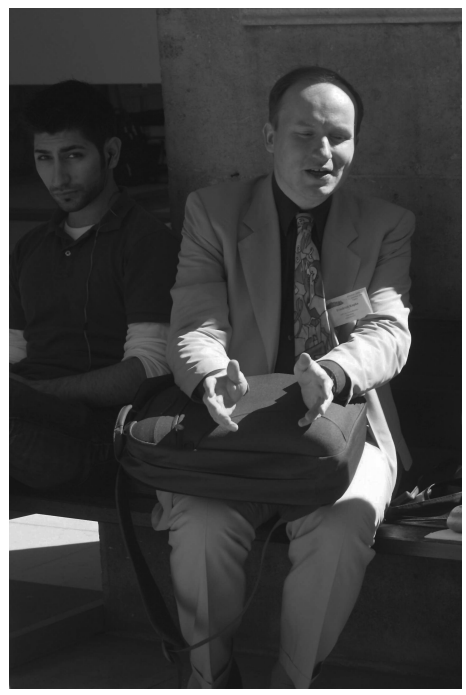

